\title{
Derivative Formula and Harnack Inequality for Degenerate Functional SDEs*
}

\author{
Jianhai Bao ${ }^{b)}$, Feng-Yu Wang ${ }^{a), b)}$, Chenggui Yuan ${ }^{b)}$ \\ ${ }^{a)}$ School of Mathematical Sciences, Beijing Normal University, Beijing 100875, China \\ ${ }^{b)}$ Department of Mathematics, Swansea University, Singleton Park, SA2 8PP, UK \\ wangfy@bnu.edu.cn, F.-Y.Wang@swansea.ac.uk, C.Yuan@swansea.ac.uk
}

October 13, 2018

\begin{abstract}
By constructing successful couplings, the derivative formula, gradient estimates and Harnack inequalities are established for the semigroup associated with a class of degenerate functional stochastic differential equations.
\end{abstract}

AMS subject Classification: 60H10, 47G20.

Keywords: Coupling, derivative formula, gradient estimate, Harnack inequality, functional stochastic differential equation.

\section{Introduction}

In recent years, the coupling argument developed in [1] for establishing dimension-free Harnack inequality in the sense of [13] has been intensively applied to the study of Markov semigroups associated with a number of stochastic (partial) differential equations, see e.g. [3, 4, 6, 7, 8, 9, 14, 16, 18, 19, 20, 22] and references within. In particular, the Harnack inequalities have been established in [4, 19] for a class of non-degenerate functional stochastic differential equations (SDEs), while the (Bismut-Elworthy-Li type) derivative formula and applications have been investigated in [5] for a class of degenerate SDEs (see also [21, 23] for the study by using Malliavin calculus). The aim of this paper is to establish the derivative formula and (log-)Harnack inequalities for degenerate functional SDEs. The derivative formula implies explicit gradient estimates of the associated semigroup, while a number of

*Supported in part by SRFDP and the Fundamental Research Funds for the Central Universities. 
applications of the (log-)Harnack inequalities have been summarized in [17, §4.2] on heat kernel estimates, entropy-cost inequalities, characterizations of invariant measures and contractivity properties of the semigroup.

Let $m \in \mathbb{Z}_{+}$and $d \in \mathbb{N}$. Denote $\mathbb{R}^{m+d}=\mathbb{R}^{m} \times \mathbb{R}^{d}$, where $\mathbb{R}^{m}=\{0\}$ when $m=0$. For $r_{0}>0$, let $\mathscr{C}:=C\left(\left[-r_{0}, 0\right] ; \mathbb{R}^{m+d}\right)$ be the space of continuous functions from $\left[-r_{0}, 0\right]$ into $\mathbb{R}^{m+d}$, which is a Banach space with the uniform norm $\|\cdot\|_{\infty}$. Consider the following functional SDE on $\mathbb{R}^{m+d}$ :

$$
\left\{\begin{array}{l}
\mathrm{d} X(t)=\{A X(t)+M Y(t)\} \mathrm{d} t \\
\mathrm{~d} Y(t)=\left\{Z(X(t), Y(t))+b\left(X_{t}, Y_{t}\right)\right\} \mathrm{d} t+\sigma \mathrm{d} B(t),
\end{array}\right.
$$

where $B(t)$ is a $d$-dimensional Brownian motion, $\sigma$ is an invertible $d \times d$-matrix, $A$ is an $m \times m$-matrix, $M$ is an $m \times d$-matrix, $Z: \mathbb{R}^{m} \times \mathbb{R}^{d} \rightarrow \mathbb{R}^{d}$ and $b: \mathscr{C} \rightarrow \mathbb{R}^{d}$ are locally Lipschitz continuous (i.e. Lipschitzian on compact sets), $\left(X_{t}, Y_{t}\right)_{t \geq 0}$ is a process on $\mathscr{C}$ with $\left(X_{t}, Y_{t}\right)(\theta):=(X(t+\theta), Y(t+\theta)), \theta \in\left[-r_{0}, 0\right]$. We assume that there exists an integer number $0 \leq k \leq m-1$ such that

$$
\operatorname{Rank}\left[M, A M, \cdots, A^{k} M\right]=m
$$

When $m=0$ this condition automatically holds by convention. Note that when $m \geq 1$, this rank condition holds for some $k>m-1$ if and only if it holds for $k=m-1$.

Let $\nabla, \nabla^{(1)}$ and $\nabla^{(2)}$ denote the gradient operators on $\mathbb{R}^{m+d}, \mathbb{R}^{m}$ and $\mathbb{R}^{d}$ respectively, and let

$$
\begin{aligned}
L f(x, y):= & \left\langle A x+M y, \nabla^{(1)} f(x, y)\right\rangle+\left\langle Z(x, y), \nabla^{(2)} f(x, y)\right\rangle \\
& +\frac{1}{2} \sum_{i, j=1}^{d}\left(\sigma \sigma^{*}\right)_{i j} \frac{\partial^{2}}{\partial y_{i} \partial y_{j}} f(x, y), \quad(x, y) \in \mathbb{R}^{m+d}, f \in C^{2}\left(\mathbb{R}^{m+d}\right) .
\end{aligned}
$$

Since both $Z$ and $b$ are locally Lipschitz continuous, due to [12] the equation (1.1) has a unique local solution for any initial data $\left(X_{0}, Y_{0}\right) \in \mathscr{C}$. To ensure the non-explosion and further regular properties of the solution, we make use of the following assumptions:

(A) There exist constants $\lambda, l>0$ and $W \in C^{2}\left(\mathbb{R}^{m+d}\right)$ of compact level sets with $W \geq 1$ such that

(A1) $L W \leq \lambda W, \quad\left|\nabla^{(2)} W\right| \leq \lambda W$

$(A 2)\left\langle b(\xi), \nabla^{(2)} W(\xi(0))\right\rangle \leq \lambda\|W(\xi)\|_{\infty}, \quad \xi \in \mathscr{C} ;$

(A3) $\left|Z(z)-Z\left(z^{\prime}\right)\right| \leq \lambda\left|z-z^{\prime}\right| W\left(z^{\prime}\right)^{l}, \quad z, z^{\prime} \in \mathbb{R}^{m+d},\left|z-z^{\prime}\right| \leq 1$

$(A 4)\left|b(\xi)-b\left(\xi^{\prime}\right)\right| \leq \lambda\left\|\xi-\xi^{\prime}\right\|_{\infty}\left\|W\left(\xi^{\prime}\right)\right\|_{\infty}^{l}, \xi, \xi^{\prime} \in \mathscr{C},\left\|\xi-\xi^{\prime}\right\|_{\infty} \leq 1$. 
Comparing with the framework investigated in [5, 23], where $b=0, A=0$ and $\operatorname{Rank}[M]=$ $m$ are assumed, the present model is more general and the segment process we are going to investigate is an infinite-dimensional Markov process. On the other hand, unlike in [5] where the condition $\left|\nabla^{(2)} W\right| \leq \lambda W$ is not used, in the present setting this condition seems essential in order to derive moment estimates of the segment process (see the proof of Lemma 2.1 below). Moreover, if $|\nabla W| \leq c W$ holds for some constant $c>0$, then $(A 3)$ and (A4) hold for some $\lambda>0$ if and only if there exists a constant $\lambda^{\prime}>0$ such that $|\nabla Z| \leq \lambda^{\prime} W^{l}$ and $|\nabla b| \leq \lambda^{\prime}\|W\|_{\infty}^{l}$ holds on $\mathbb{R}^{m+d}$ and $\mathscr{C}$ respectively.

It is easy to see that (A) holds for $W(z)=1+|z|^{2}, l=1$ and some constant $\lambda>0$ provided that $Z$ and $b$ are globally Lipschitz continuous on $\mathbb{R}^{m+d}$ and $\mathscr{C}$ respectively. It is clear that $(A 1)$ and $(A 2)$ imply the non-explosion of the solution (see Lemma 2.1 below). In this paper we aim to investigate regularity properties of the Markov semigroup associated with the segment process:

$$
P_{t} f(\xi)=\mathbb{E}^{\xi} f\left(X_{t}, Y_{t}\right), \quad f \in \mathscr{B}_{b}(\mathscr{C}), \xi \in \mathscr{C},
$$

where $\mathscr{B}_{b}(\mathscr{C})$ is the class of all bounded measurable functions on $\mathscr{C}$ and $\mathbb{E}^{\xi}$ stands for the expectation for the solution starting at the point $\xi \in \mathscr{C}$. When $m=0$ we have $X_{t} \equiv 0$ and $\mathscr{C}=\{0\} \times \mathscr{C}_{2} \equiv \mathscr{C}_{2}:=C\left(\left[-r_{0}, 0\right] ; \mathbb{R}^{d}\right)$, so that $P_{t} f$ can be simply formulated as $P_{t} f(\xi)=\mathbb{E}^{\xi} f\left(Y_{t}\right)$ for $f \in \mathscr{B}_{b}\left(\mathscr{C}_{2}\right), \xi \in \mathscr{C}_{2}$. Thus, (1.1) also includes non-degenerate functional SDEs. For any $h=\left(h_{1}, h_{2}\right) \in \mathscr{C}$ and $z \in \mathbb{R}^{m+d}$, let $\nabla_{h}$ and $\nabla_{z}$ be the directional derivatives along $h$ and $z$ respectively. The following result provides an explicit derivative formula for $P_{T}, T>r_{0}$.

T1.1 Theorem 1.1. Assume $(\mathbf{A})$ and let $T>r_{0}$. Let $v:[0, T] \rightarrow \mathbb{R}$ and $\alpha:[0, T] \rightarrow \mathbb{R}^{m}$ be Lipschitz continuous such that $v(0)=1, \alpha(0)=0, v(s)=0, \alpha(s)=0$ for $s \geq T-r_{0}$, and

$$
h_{1}(0)+\int_{0}^{t} \mathrm{e}^{-s A} M \phi(s) \mathrm{d} s=0, \quad t \geq T-r_{0},
$$

where $\phi(s):=v(s) h_{2}(0)+\alpha(s)$. Then for any $h=\left(h_{1}, h_{2}\right) \in \mathscr{C}$ and $f \in \mathscr{B}_{b}(\mathscr{C})$,

$$
\nabla_{h} P_{T} f(\xi)=\mathbb{E}^{\xi}\left\{f\left(X_{T}, Y_{T}\right) \int_{0}^{T}\left\langle N(s),\left(\sigma^{*}\right)^{-1} \mathrm{~d} B(s)\right\rangle\right\}, \quad \xi \in \mathscr{C}
$$

holds for

$$
N(s):=\left(\nabla_{\Theta(s)} Z\right)(X(s), Y(s))+\left(\nabla_{\Theta_{s}} b\right)\left(X_{s}, Y_{s}\right)-v^{\prime}(s) h_{2}(0)-\alpha^{\prime}(s), \quad s \in[0, T],
$$

where

$$
\Theta(s)=\left(\Theta^{(1)}(s), \Theta^{(2)}(s)\right):= \begin{cases}h(s), & \text { if } s \leq 0 \\ \left(\mathrm{e}^{A s} h_{1}(0)+\int_{0}^{s} \mathrm{e}^{(s-r) A} M \phi(r) \mathrm{d} r, \phi(s)\right), & \text { if } s>0 .\end{cases}
$$


A simple choice of $v$ is

$$
v(s)=\frac{\left(T-r_{0}-s\right)^{+}}{T-r_{0}}, \quad s \geq 0 .
$$

To present a specific choice of $\alpha$, let

$$
Q_{t}:=\int_{0}^{t} \frac{s\left(T-r_{0}-s\right)^{+}}{\left(T-r_{0}\right)^{2}} \mathrm{e}^{-s A} M M^{*} \mathrm{e}^{-s A^{*}} \mathrm{~d} s, \quad t>0 .
$$

According to [11] (see also [21, Proof of Theorem 4.2(1)]), when $m \geq 1$ the matrix $Q_{t}$ is invertible with

$$
\left\|Q_{t}^{-1}\right\| \leq c\left(T-r_{0}\right)(t \wedge 1)^{-2(k+1)}, \quad t>0
$$

for some constant $c>0$.

C1.2 Corollary 1.2. Assume (A) and let $T>r_{0}$. Then (1.4) holds for $v(s)=\frac{\left(T-r_{0}-s\right)^{+}}{T-r_{0}}$ and

$$
\alpha(s)=-\frac{s\left(T-r_{0}-s\right)^{+}}{\left(T-r_{0}\right)^{2}} M^{*} \mathrm{e}^{-s A^{*}} Q_{T-r_{0}}^{-1}\left(h_{1}(0)+\int_{0}^{T-r_{0}} \frac{\left(T-r_{0}-r\right)^{+}}{T-r_{0}} \mathrm{e}^{-r A} M h_{2}(0) \mathrm{d} r\right),
$$

where by convention $M=0$ (hence, $\alpha=0$ ) if $m=0$.

The following gradient estimates are direct consequences of Theorem 1.1.

C1.3 Corollary 1.3. Assume (A). Then:

(1) There exists a constant $C \in(0, \infty)$ such that

$$
\begin{aligned}
\left|\nabla_{h} P_{T} f(\xi)\right| \leq C & \sqrt{P_{T} f^{2}(\xi)}\left\{|h(0)|\left(1+\frac{\|M\|}{\left(T-r_{0}\right)^{2 k+1} \wedge 1}\right)\right. \\
& \left.+\|W(\xi)\|_{\infty}^{l} \sqrt{T \wedge\left(1+r_{0}\right)}\left(\|h\|_{\infty}+\frac{\|M\| \cdot|h(0)|}{\left(T-r_{0}\right)^{2 k+1} \wedge 1}\right)\right\}
\end{aligned}
$$

holds for all $T>r_{0}, \xi, h \in \mathscr{C}$ and $f \in \mathscr{B}_{b}(\mathscr{C})$;

(2) Let $\left|\nabla^{(2)} W\right|^{2} \leq \delta W$ hold for some constant $\delta>0$. If $l \in[0,1 / 2)$ then there exists a constant $C \in(0, \infty)$ such that

$$
\begin{aligned}
& \left|\nabla_{h} P_{T} f(\xi)\right| \leq r\left\{P_{T} f \log f-\left(P_{T} f\right) \log P_{T} f\right\}(\xi) \\
& \quad+\frac{C P_{T} f(\xi)}{r}\left\{|h(0)|^{2}\left(\frac{1}{\left(T-r_{0}\right) \wedge 1}+\frac{\|M\|^{2}}{\left\{\left(T-r_{0}\right) \wedge 1\right\}^{4 k+3}}\right)\right. \\
& \left.\quad+\|h\|_{\infty}^{2}\|W(\xi)\|_{\infty}+\left(\|h\|_{\infty}^{2}+\frac{|h(0)|^{2}\|M\|^{2}}{\left\{\left(T-r_{0}\right) \wedge 1\right\}^{4 k+2}}\right)^{\frac{1}{1-2 l}}\left(\frac{r^{2}}{\|h\|_{\infty}^{2}} \vee 1\right)^{\frac{2 l}{1-2 l}}\right\}
\end{aligned}
$$

holds for all $r>0, T>r_{0}, \xi, h \in \mathscr{C}$ and positive $f \in \mathscr{B}_{b}(\mathscr{C})$; 
(3) Let $\left|\nabla^{(2)} W\right|^{2} \leq \delta W$ hold for some constant $\delta>0$. If $l=\frac{1}{2}$ then there exist constants $C, C^{\prime} \in(0, \infty)$ such that

$$
\begin{aligned}
\left|\nabla_{h} P_{T} f(\xi)\right| \leq & r\left\{P_{T} f \log f-\left(P_{T} f\right) \log P_{T} f\right\}(\xi) \\
& +\frac{C P_{T} f(\xi)}{r}\left\{|h(0)|^{2}\left(\frac{1}{\left(T-r_{0}\right) \wedge 1}+\frac{\|M\|^{2}}{\left\{\left(T-r_{0}\right) \wedge 1\right\}^{4 k+3}}\right)\right. \\
& \left.+\|W(\xi)\|_{\infty}\left(\|h\|_{\infty}^{2}+\frac{\|M\|^{2}|h(0)|^{2}}{\left\{\left(T-r_{0}\right) \wedge 1\right\}^{4 k+2}}\right)\right\}
\end{aligned}
$$

holds for

$$
r \geq C^{\prime}\left(\|h\|_{\infty}+\frac{\|M\| \cdot|h(0)|}{\left\{\left(T-r_{0}\right) \wedge 1\right\}^{2 k+1}}\right),
$$

all $T>r_{0}, \xi, h \in \mathscr{C}$ and positive $f \in \mathscr{B}_{b}(\mathscr{C})$.

When $m=0$ the above assertions hold with $\|M\|=0$.

According to [2], the entropy gradient estimate implies the Harnack inequality with power, we have the following result which follows immediately from Corollary 1.3 (2) and [5, Proposition 4.1]. Similarly, Corollary 1.3 (3) implies the same type Harnack inequality for smaller $\|h\|_{\infty}$ comparing to $T-r_{0}$.

C1.4 Corollary 1.4. Assume (A) and let $\left|\nabla^{(2)} W\right|^{2} \leq \delta W$ hold for some constant $\delta>0$. If $l \in\left[0, \frac{1}{2}\right)$ then there exists a constant $C \in(0, \infty)$ such that

$$
\begin{aligned}
\left(P_{T} f\right)^{p}(\xi+h) \leq P_{T} & f^{p}(\xi) \exp \left[\frac { C p } { p - 1 } \left\{\|h\|_{\infty}^{2} \int_{0}^{1}\|W(\xi+s h)\|_{\infty} \mathrm{d} s\right.\right. \\
& \left.\left.+\left(\|h\|_{\infty}^{2}+\frac{\|M\|^{2}|h(0)|^{2}}{\left\{\left(T-r_{0}\right) \wedge 1\right\}^{4 k+2}}\right)^{\frac{1}{1-2 l}}\left(\frac{(p-1)^{2}}{\|h\|_{\infty}^{2}} \vee 1\right)^{\frac{2 l}{1-2 l}}\right\}\right]
\end{aligned}
$$

holds for all $T>r_{0}, p>1, \xi, h \in \mathscr{C}$ and positive $f \in \mathscr{B}_{b}(\mathscr{C})$. If $m=0$ then the assertion holds for $\|M\|=0$.

Finally, we consider the log-Harnack inequality introduced in [10, 15]. To this end, as in [5], we slightly strengthen $(A 3)$ and $(A 4)$ as for follows: there exists an increasing function $U$ on $[0, \infty)$ such that

$$
\begin{aligned}
& \left|Z(z)-Z\left(z^{\prime}\right)\right| \leq \lambda\left|z-z^{\prime}\right|\left\{W\left(z^{\prime}\right)^{l}+U\left(\left|z-z^{\prime}\right|\right)\right\}, \quad z, z^{\prime} \in \mathbb{R}^{m+d} \\
& \left|b(\xi)-b\left(\xi^{\prime}\right)\right| \leq \lambda\left\|\xi-\xi^{\prime}\right\|_{\infty}\left\{\left\|W\left(\xi^{\prime}\right)\right\|_{\infty}^{l}+U\left(\left\|\xi-\xi^{\prime}\right\|_{\infty}\right)\right\}, \xi, \xi^{\prime} \in \mathscr{C} .
\end{aligned}
$$

Obviously, if

$$
W(z)^{l} \leq c\left\{W\left(z^{\prime}\right)^{l}+U\left(\left|z-z^{\prime}\right|\right)\right\}, \quad z, z^{\prime} \in \mathbb{R}^{m+d}
$$

holds for some constant $c>0$, then $(A 3)$ and $(A 4)$ imply $\left(A 3^{\prime}\right)$ and $\left(A 4^{\prime}\right)$ respectively with possibly different $\lambda$. 
T1.5 Theorem 1.5. Assume $(A 1),(A 2),\left(A 3^{\prime}\right)$ and $\left(A 4^{\prime}\right)$. Then there exists a constant $C \in$ $(0, \infty)$ such that for any positive $f \in \mathscr{B}_{b}(\mathscr{C}), T>r_{0}$ and $\xi, h \in \mathscr{C}$,

$$
\begin{aligned}
P_{T} \log f(\xi+h)-\log P_{T} f(\xi) \leq C\{ & {\left[\|W(\xi+h)\|_{\infty}^{2 l}+U^{2}\left(C\|h\|_{\infty}+\frac{C\|M\| \cdot|h(0)|}{\left(T-r_{0}\right) \wedge 1}\right)\right]\|h\|_{\infty}^{2} } \\
& \left.+\frac{|h(0)|^{2}}{\left(T-r_{0}\right) \wedge 1}+\frac{\|M\|^{2}|h(0)|^{2}}{\left\{\left(T-r_{0}\right) \wedge 1\right\}^{4 k+3}}\right\} .
\end{aligned}
$$

If $m=0$ then the assertion holds for $\|M\|=0$.

For applications of the Harnack and log-Harnack inequalities we are referred to [17, §4.2]. The remainder of the paper is organized as follows: Theorem 1.1 and Corollary 1.2 are proved Section 2, while Corollary 1.3 and Theorem 1.5 are proved in Section 3; in Section 4 the assumption (A) is weakened for the discrete time delay case, and two examples are presented to illustrate our results.

\section{Proofs of Theorem 1.1 and Corollary 1.2}

lem1 Lemma 2.1. Assume $(A 1)$ and $(A 2)$. Then for any $k>0$ there exists a constant $C>0$ such that

$$
\mathbb{E}^{\xi} \sup _{-r_{0} \leq s \leq t} W(X(s), Y(s))^{k} \leq 3\|W(\xi)\|_{\infty}^{k} \mathrm{e}^{C t}, \quad t \geq 0, \quad \xi \in \mathscr{C}
$$

holds. Consequently, the solution is non-explosive.

Proof. For any $n \geq 1$, let

$$
\tau_{n}:=\inf \{t \in[0, T]:|X(t)|+|Y(t)| \geq n\} .
$$

Moreover, let

$$
\ell(s):=W(X, Y)(s), \quad s \geq-r_{0} .
$$

By the Itô formula and using the first inequality in $(A 1)$ and $(A 2)$ we may find a constant $C_{1}>0$ such that

W2 (2.1)

$$
\begin{aligned}
\ell(t & \left.\wedge \tau_{n}\right)^{k}=\ell(0)^{k}+k \int_{0}^{t \wedge \tau_{n}} \ell(s)^{k-1}\left\langle\nabla^{(2)} W(X, Y)(s), \sigma \mathrm{d} B(s)\right\rangle \\
& +k \int_{0}^{t \wedge \tau_{n}} \ell(s)^{k-1}\left\{L W(X, Y)(s)+\left\langle b\left(X_{s}, Y_{s}\right), \nabla^{(2)} W(X, Y)(s)\right\rangle\right. \\
& \left.+\frac{1}{2}(k-1) \ell(s)^{-1}\left|\sigma^{*} \nabla^{(2)} W(X, Y)(s)\right|^{2}\right\} \mathrm{d} s \\
\leq & l(0)^{k}+k \int_{0}^{t \wedge \tau_{n}} \ell(s)^{k-1}\left\langle\nabla^{(2)} W(X, Y)(s), \sigma \mathrm{d} B(s)\right\rangle+C_{1} \int_{0}^{t \wedge \tau_{n}} \sup _{r \in\left[-r_{0}, s\right]} \ell(r)^{k} \mathrm{~d} s .
\end{aligned}
$$

Noting that by the second inequality in $(A 1)$ and the Burkholder-Davis-Gundy inequality we obtain 


$$
\begin{aligned}
& k \mathbb{E}^{\xi} \sup _{s \in[0, t]}\left|\int_{0}^{s \wedge \tau_{n}} \ell(r)^{k-1}\left\langle\nabla^{(2)} W(X, Y)(s), \sigma \mathrm{d} B(r)\right\rangle\right| \leq C_{2} \mathbb{E}^{\xi}\left(\int_{0}^{t} \ell\left(s \wedge \tau_{n}\right)^{2 k} \mathrm{~d} s\right)^{1 / 2} \\
& \leq C_{2} \mathbb{E}^{\xi}\left\{\left(\sup _{s \in[0, t]} \ell\left(s \wedge \tau_{n}\right)^{k}\right)^{1 / 2}\left(\int_{0}^{t} \ell\left(s \wedge \tau_{n}\right)^{k} \mathrm{~d} s\right)^{1 / 2}\right\} \\
& \leq \frac{1}{2} \mathbb{E}^{\xi} \sup _{s \in[0, t]} \ell\left(s \wedge \tau_{n}\right)^{k}+\frac{C_{2}^{2}}{2} \mathbb{E}^{\xi} \int_{0}^{t} \sup _{r \in[0, s]} \ell\left(r \wedge \tau_{n}\right)^{k} \mathrm{~d} s
\end{aligned}
$$

for some constant $C_{2}>0$. Combining this with (2.1) and noting that $\left(X_{0}, Y_{0}\right)=\xi$, we conclude that there exists a constant $C>0$ such that

$$
\mathbb{E}^{\xi} \sup _{-r_{0} \leq s \leq t} \ell\left(s \wedge \tau_{n}\right)^{k} \leq 3\|W(\xi)\|_{\infty}^{k}+C \mathbb{E}^{\xi} \int_{0}^{t} \sup _{s \in\left[-r_{0}, t\right]} \ell(s)^{k} \mathrm{~d} s, \quad t \geq 0 .
$$

Due to the Gronwall lemma this implies that

$$
\mathbb{E}^{\xi} \sup _{-r_{0} \leq s \leq t} \ell\left(s \wedge \tau_{n}\right)^{k} \leq 3\|W(\xi)\|_{\infty}^{k} \mathrm{e}^{C t}, \quad t \geq 0, n \geq 1
$$

Consequently, we have $\tau_{n} \uparrow \infty$ as $n \uparrow \infty$, and thus the desired inequality follows by letting $n \rightarrow \infty$.

To establish the derivative formula, we first construct couplings for solutions starting from $\xi$ and $\xi+\varepsilon h$ for $\varepsilon \in(0,1]$, then let $\varepsilon \rightarrow 0$. For fixed $\xi=\left(\xi_{1}, \xi_{2}\right), h=\left(h_{1}, h_{2}\right) \in \mathscr{C}$, let $(X(t), Y(t))$ solve (1.1) with $\left(X_{0}, Y_{0}\right)=\xi$; and for any $\varepsilon \in(0,1]$, let $\left(X^{\varepsilon}(t), Y^{\varepsilon}(t)\right)$ solve the equation

$$
\left\{\begin{array}{l}
\mathrm{d} X^{\varepsilon}(t)=\left\{A X^{\varepsilon}(t)+M Y^{\varepsilon}(t)\right\} \mathrm{d} t, \\
\mathrm{~d} Y^{\varepsilon}(t)=\left\{Z(X(t), Y(t))+b\left(X_{t}, Y_{t}\right)\right\} \mathrm{d} t+\sigma \mathrm{d} B(t)+\varepsilon\left\{v^{\prime}(t) h_{2}(0)+\alpha^{\prime}(t)\right\} \mathrm{d} t
\end{array}\right.
$$

with $\left(X_{0}^{\varepsilon}, Y_{0}^{\varepsilon}\right)=\xi+\varepsilon h$. By Lemma 2.1 and (2.3) below, the solution to (2.2) is non-explosive as well.

Pro1 Proposition 2.2. Let $\phi(s):=v(s) h_{2}(0)+\alpha(s), s \in[0, T]$, and the conditions of Theorem 1.1 hold. Then

$$
\left(X^{\varepsilon}(t), Y^{\varepsilon}(t)\right)=(X(t), Y(t))+\varepsilon \Theta(t), \quad \varepsilon, t \geq 0
$$

holds for

$$
\Theta(t):=\left(\Theta^{(1)}(t), \Theta^{(2)}(t)\right):= \begin{cases}h(t), & \text { if } t \leq 0, \\ \left(\mathrm{e}^{A t} h_{1}(0)+\int_{0}^{t} \mathrm{e}^{(t-r) A} M \phi(r) \mathrm{d} r, \phi(t)\right), & \text { if } t>0 .\end{cases}
$$

In particular, $\left(X_{T}^{\varepsilon}, Y_{T}^{\varepsilon}\right)=\left(X_{T}, Y_{T}\right)$. 
Proof. By (2.2) and noting that $v(0)=1$ and $v(s)=0$ for $s \geq T-r_{0}$, we have $Y^{\varepsilon}(t)=$ $Y(t)+\varepsilon \phi(t)$ and

$$
X^{\varepsilon}(t)=X(t)+\varepsilon \mathrm{e}^{A t} h_{1}(0)+\varepsilon \int_{0}^{t} \mathrm{e}^{(t-s) A} M \phi(s) \mathrm{d} s, \quad t \geq 0 .
$$

Thus, (2.3) holds. Moreover, since $\alpha(s)=v(s)=0$ for $s \geq T-r_{0}$, we have $\Theta^{(2)}(s)=\phi(s)=0$ for $s \geq T-r_{0}$. Moreover, by (1.3) we have $\Theta^{(1)}(s)=0$ for $s \geq T-r_{0}$. Therefore, the proof is finished.

Since according to Proposition 2.2 we have $\left(X_{T}^{\varepsilon}, Y_{T}^{\varepsilon}\right)=\left(X_{T}, Y_{T}\right)$. Noting that $\left(X_{0}^{\varepsilon}, Y_{0}^{\varepsilon}\right)=$ $\xi+\varepsilon h$, if (2.2) can be formulated as (1.1) using a different Brownian motion, then we are able to link $P_{T} f(\xi)$ to $P_{T} f(\xi+\varepsilon h)$ and furthermore derive the derivative formula by taking derivative w.r.t. $\varepsilon$ at $\varepsilon=0$. To this end, let

$$
\Phi^{\varepsilon}(s)=Z(X(s), Y(s))-Z\left(X^{\varepsilon}(s), Y^{\varepsilon}(s)\right)+b\left(X_{s}, Y_{s}\right)-b\left(X_{s}^{\varepsilon}, Y_{s}^{\varepsilon}\right)+\varepsilon\left\{v^{\prime}(s) h_{2}(0)+\alpha^{\prime}(s)\right\} .
$$

Set

$$
R^{\varepsilon}(s)=\exp \left[-\int_{0}^{s}\left\langle\sigma^{-1} \Phi^{\varepsilon}(r), \mathrm{d} B(r)\right\rangle-\frac{1}{2} \int_{0}^{s}\left|\sigma^{-1} \Phi^{\varepsilon}(r)\right|^{2} \mathrm{~d} r\right]
$$

and

$$
B^{\varepsilon}(s)=B(s)+\int_{0}^{s} \sigma^{-1} \Phi^{\varepsilon}(r) \mathrm{d} r
$$

Then (2.2) reduces to

$$
\left\{\begin{array}{l}
\mathrm{d} X^{\varepsilon}(t)=\left\{A X^{\varepsilon}(t)+M Y^{\varepsilon}(t)\right\} \mathrm{d} t \\
\mathrm{~d} Y^{\varepsilon}(t)=\left\{Z\left(X^{\varepsilon}(t), Y^{\varepsilon}(t)\right)+b\left(X_{t}^{\varepsilon}, Y_{t}^{\varepsilon}\right)\right\} \mathrm{d} t+\sigma \mathrm{d} B^{\varepsilon}(t) .
\end{array}\right.
$$

According to the Girsanov theorem, to ensure that $B^{\varepsilon}(t)$ is a Browanian motion under $\mathbb{Q}_{\varepsilon}:=R^{\varepsilon}(T) \mathbb{P}$, we first prove that $R^{\varepsilon}(t)$ is an exponential martingale. Moreover, to obtain the derivative formula using the dominated convergence theorem, we also need $\left\{\frac{R^{\varepsilon}(T)-1}{\varepsilon}\right\}_{\varepsilon \in(0,1)}$ to be uniformly integrable. Therefore, we will need the following two lemmas.

L2.2 Lemma 2.3. Let (A) hold. Then there exists $\varepsilon_{0}>0$ such that

$$
\sup _{s \in[0, T], \varepsilon \in\left(0, \varepsilon_{0}\right)} \mathbb{E}\left[R^{\varepsilon}(s) \log R^{\varepsilon}(s)\right]<\infty,
$$

so that for each $\varepsilon \in(0,1),\left(R^{\varepsilon}(s)\right)_{s \in[0, T]}$ is a uniformly integrable martingale.

Proof. By (2.3), there exists $\varepsilon_{0}>0$ such that

$$
\varepsilon_{0}|\Theta(t)| \leq 1, \quad t \in\left[-r_{0}, T\right]
$$

For any $\varepsilon \in\left[0, \varepsilon_{0}\right]$, define

$$
\tau_{n}:=\inf \left\{t \geq 0:|X(t)|+|Y(t)|+\left|X^{\varepsilon}(t)\right|+\left|Y^{\varepsilon}(t)\right| \geq n\right\}, n \geq 1 .
$$


We have $\tau_{n} \uparrow \infty$ as $n \uparrow \infty$ due to the non-explosion. By the Girsanov theorem, the process $\left\{R^{\varepsilon}\left(s \wedge \tau_{n}\right)\right\}_{s \in[0, T]}$ is a martingale and $\left\{B^{\varepsilon}(s)\right\}_{s \in\left[0, T \wedge \tau_{n}\right]}$ is a Brownian motion under the probability measure $\mathbb{Q}_{\varepsilon, n}:=R^{\varepsilon}\left(T \wedge \tau_{n}\right) \mathbb{P}$. By the definition of $R^{\varepsilon}(s)$ we have

$$
\mathbb{E}\left[R^{\varepsilon}\left(s \wedge \tau_{n}\right) \log R^{\varepsilon}\left(s \wedge \tau_{n}\right)\right]=\mathbb{E}_{\mathbb{Q}_{\varepsilon, n}}\left[\log R^{\varepsilon}\left(s \wedge \tau_{n}\right)\right] \leq \frac{1}{2} \mathbb{E}_{\mathbb{Q}_{\varepsilon, n}} \int_{0}^{T \wedge \tau_{n}}\left|\sigma^{-1} \Phi^{\varepsilon}(r)\right|^{2} \mathrm{~d} r .
$$

By (2.5), (A3) and $(A 4)$,

$$
\left|\sigma^{-1} \Phi^{\varepsilon}(s)\right|^{2} \leq c \varepsilon^{2}\left\|W\left(X_{s}^{\varepsilon}, Y_{s}^{\varepsilon}\right)\right\|_{\infty}^{2 l},
$$

holds for some constant $c$ independent of $\varepsilon$. By the weak uniqueness of the solution to (1.1) and (2.4), the distribution of $\left(X^{\varepsilon}(s), Y^{\varepsilon}(s)\right)_{s \in\left[0, T \wedge \tau_{n}\right]}$ under $\mathbb{Q}_{\varepsilon, n}$ coincides with that of the solution to (1.1) with $\left(X_{0}, Y_{0}\right)=\xi+\varepsilon h$ up to time $T \wedge \tau_{n}$, we therefore obtain from Lemma 2.1 that

$$
\mathbb{E}\left[R^{\varepsilon}\left(s \wedge \tau_{n}\right) \log R^{\varepsilon}\left(s \wedge \tau_{n}\right)\right] \leq c\|W(\xi+\varepsilon h)\|_{\infty}^{2 l} \int_{0}^{T} \mathrm{e}^{C t} \mathrm{~d} t<\infty, \quad n \geq 1, \varepsilon \in\left(0, \varepsilon_{0}\right) .
$$

Then the required assertion follows by letting $n \rightarrow \infty$.

L2.3 Lemma 2.4. If (A) holds, then there exists $\varepsilon_{0}>0$ such that

$$
\sup _{\varepsilon \in\left(0, \varepsilon_{0}\right)} \mathbb{E}\left(\frac{R^{\varepsilon}(T)-1}{\varepsilon} \log \frac{R^{\varepsilon}(T)-1}{\varepsilon}\right)<\infty
$$

Moreover,

$\mathrm{y} 2$

$$
\begin{aligned}
& \lim _{\varepsilon \rightarrow 0} \frac{R^{\varepsilon}(T)-1}{\varepsilon}= \\
& \int_{0}^{T}\left\langle\left(\nabla_{\Theta(s)} Z\right)(X(s), Y(s))+\left(\nabla_{\Theta_{s}} b\right)\left(X_{s}, Y_{s}\right)-v^{\prime}(s) h_{2}(0)-\alpha^{\prime}(s),\left(\sigma^{*}\right)^{-1} \mathrm{~d} B(s)\right\rangle .
\end{aligned}
$$

Proof. Let $\varepsilon_{0}$ be such that (2.5) holds. Since (2.8) is a direct consequence of (2.3) and the definition of $R^{\varepsilon}(T)$, we only prove the first assertion. By [5] we know that

$$
\frac{R^{\varepsilon}(T)-1}{\varepsilon} \log \frac{R^{\varepsilon}(T)-1}{\varepsilon} \leq 2 R^{\varepsilon}(T)\left(\frac{\log R^{\varepsilon}(T)}{\varepsilon}\right)^{2} .
$$

Since due to Lemma $2.3\left\{B^{\varepsilon}(t)\right\}_{t \in[0, T]}$ is a Brownian motion under the probability measure $\mathbb{Q}_{\varepsilon}:=R^{\varepsilon}(T) \mathbb{P}$, and since

$$
\begin{aligned}
\log R^{\varepsilon}(T) & =-\int_{0}^{T}\left\langle\sigma^{-1} \Phi^{\varepsilon}(r), \mathrm{d} B(r)\right\rangle-\frac{1}{2} \int_{0}^{T}\left|\sigma^{-1} \Phi^{\varepsilon}(r)\right|^{2} \mathrm{~d} r \\
& =-\int_{0}^{T}\left\langle\sigma^{-1} \Phi^{\varepsilon}(r), \mathrm{d} B^{\varepsilon}(r)\right\rangle+\frac{1}{2} \int_{0}^{T}\left|\sigma^{-1} \Phi^{\varepsilon}(r)\right|^{2} \mathrm{~d} r
\end{aligned}
$$


it follows from (2.7) that

$$
\begin{aligned}
& \mathbb{E}\left(\frac{R^{\varepsilon}(T)-1}{\varepsilon} \log \frac{R^{\varepsilon}(T)-1}{\varepsilon}\right) \leq \mathbb{E}\left(2 R^{\varepsilon}(T)\left(\frac{\log R^{\varepsilon}(T)}{\varepsilon}\right)^{2}\right)=2 \mathbb{E}_{\mathbb{Q}_{\varepsilon}}\left(\frac{\log R^{\varepsilon}(T)}{\varepsilon}\right)^{2} \\
& \leq \frac{4}{\varepsilon^{2}} \mathbb{E}_{\mathbb{Q}_{\varepsilon}}\left(\int_{0}^{T}\left\langle\sigma^{-1} \Phi^{\varepsilon}(r), \mathrm{d} B^{\varepsilon}(r)\right\rangle\right)^{2}+\frac{1}{\varepsilon^{2}} \mathbb{E}_{\mathbb{Q}_{\varepsilon}}\left(\int_{0}^{T}\left|\sigma^{-1} \Phi^{\varepsilon}(r)\right|^{2} \mathrm{~d} r\right)^{2} \\
& \leq \frac{4}{\varepsilon^{2}} \int_{0}^{T} \mathbb{E}_{\mathbb{Q}_{\varepsilon}}\left|\sigma^{-1} \Phi^{\varepsilon}(r)\right|^{2} \mathrm{~d} r+\frac{T}{\varepsilon^{2}} \int_{0}^{T} \mathbb{E}_{\mathbb{Q}_{\varepsilon}}\left|\sigma^{-1} \Phi^{\varepsilon}(r)\right|^{4} \mathrm{~d} r \\
& \leq c \int_{0}^{T} \mathbb{E}_{\mathbb{Q}_{\varepsilon}}\left\|W\left(X_{r}^{\varepsilon}, Y_{r}^{\varepsilon}\right)\right\|_{\infty}^{4 l} \mathrm{~d} r
\end{aligned}
$$

holds for some constant $c>0$. As explained in the proof of Lemma 2.3 the distribution of $\left(X_{s}^{\varepsilon}, Y_{s}^{\varepsilon}\right)_{s \in[0, T]}$ under $\mathbb{Q}_{\varepsilon}$ coincides with that of the segment process of the solution to (1.1) with $\left(X_{0}, Y_{0}\right)=\xi+\varepsilon h$, the first assertion follows by Lemma 2.1.

Proof of Theorem 1.1. Since Lemma 2.3, together with the Girsanov theorem, implies that $\left\{B^{\varepsilon}(s)\right\}_{s \in[0, T]}$ is a Brownian motion with respect to $\mathbb{Q}_{\varepsilon}:=R^{\varepsilon}(T) \mathbb{P}$, by (2.4) and $\left(X_{T}, Y_{T}\right)=$ $\left(X_{T}^{\varepsilon}, Y_{T}^{\varepsilon}\right)$ we obtain

$$
P_{T} f(\xi+\varepsilon h)=\mathbb{E}_{\mathbb{Q}_{\varepsilon}} f\left(X_{T}^{\varepsilon}, Y_{T}^{\varepsilon}\right)=\mathbb{E}\left\{R^{\varepsilon}(T) f\left(X_{T}, Y_{T}\right)\right\} .
$$

Thus,

$$
P_{T} f(\xi+\varepsilon h)-P_{T} f(\xi)=\mathbb{E} R^{\varepsilon}(T) f\left(X_{T}, Y_{T}\right)-\mathbb{E} f\left(X_{T}, Y_{T}\right)=\mathbb{E}\left[\left(R^{\varepsilon}(T)-1\right) f\left(X_{T}, Y_{T}\right)\right] .
$$

Combining this with Lemma 2.4 and using the dominated convergence theorem, we arrive at

$$
\begin{aligned}
\nabla_{h} P_{T} f(\xi, \eta) & =\lim _{\varepsilon \rightarrow 0} \frac{P_{T} f(\xi+\varepsilon h)-P_{T} f(\xi)}{\varepsilon}=\lim _{\varepsilon \rightarrow 0} \frac{\mathbb{E}\left[\left(R^{\varepsilon}(T)-1\right) f\left(X_{T}, Y_{T}\right)\right]}{\varepsilon} \\
& =\mathbb{E}\left\{f\left(X_{T}, Y_{T}\right) \int_{0}^{T}\left\langle N(s),\left(\sigma^{*}\right)^{-1} \mathrm{~d} B(s)\right\rangle\right\} .
\end{aligned}
$$

Proof of Corollary 1.2. It suffices to verify (1.3) for the specific $v$ and $\alpha$. Since when $m=0$ we have $h_{1}=M=0$ so that (1.3) trivially holds, we only consider $m \geq 1$. In this case, (1.3) is satisfied since according to the definition of $\phi(s)$ and $\alpha(s)$ we have for $t \geq T-r_{0}$,

$$
\begin{aligned}
& \int_{0}^{t} \mathrm{e}^{-s A} M \phi(s) \mathrm{d} s=\int_{0}^{T-r_{0}} \mathrm{e}^{-s A} M \phi(s) \mathrm{d} s \\
& =\int_{0}^{T-r_{0}} v(s) \mathrm{e}^{-s A} M h_{2}(0) \mathrm{d} s-Q_{T-r_{0}} Q_{T-r_{0}}^{-1}\left(h_{1}(0)+\int_{0}^{T-r_{0}} v(s) \mathrm{e}^{-s A} M h_{2}(0) \mathrm{d} s\right) \\
& =-h_{1}(0) .
\end{aligned}
$$




\section{Proofs of Corollary 1.3 and Theorem 1.5}

To prove the entropy-gradient estimates in Corollary (2) and (3), we need the following simple lemma which seems new and might be interesting by itself.

L3.1 Lemma 3.1. Let $\ell(t)$ be a non-negative continuous semi-martingale and let $\mathscr{M}(t)$ be a continuous martingale with $\mathscr{M}(0)=0$ such that

$$
\mathrm{d} \ell(t) \leq \mathrm{d} \mathscr{M}(t)+c \bar{\ell}_{t} \mathrm{~d} t,
$$

where $c \geq 0$ is a constant and $\bar{\ell}_{t}:=\sup _{s \in[0, t]} \ell(s)$. Then

$$
\mathbb{E} \exp \left[\frac{\varepsilon}{T \mathrm{e}^{1+c T}} \int_{0}^{T} \bar{\ell}_{t} \mathrm{~d} t\right] \leq \mathrm{e}^{\varepsilon \ell(0)+1}\left(\mathbb{E} \mathrm{e}^{2 \varepsilon^{2}\langle\mathscr{M}\rangle(T)}\right)^{1 / 2}, \quad T, \varepsilon \geq 0 .
$$

Proof. Let $\overline{\mathscr{M}}_{t}:=\sup _{s \in[0, t]} \mathscr{M}(t)$. We have

$$
\overline{\mathscr{M}}_{t}+c \int_{0}^{t} \bar{\ell}_{s} \mathrm{~d} s \geq \bar{\ell}_{t}-\ell(0) .
$$

Thus,

$$
\begin{aligned}
& \quad \frac{\ell_{T}}{\mathrm{e}^{1+c T}}-\ell(0) \leq \frac{\overline{\mathscr{M}}_{T}+c \int_{0}^{T} \bar{\ell}_{t} \mathrm{~d} t}{\mathrm{e}^{1+c T}}-\left(1-\mathrm{e}^{-(1+c T)}\right) \ell(0) \\
& =\int_{0}^{T} \mathrm{~d}\left\{\mathrm{e}^{-\left(c+T^{-1}\right) t}\left(\overline{\mathscr{M}}_{t}+c \int_{0}^{t} \bar{\ell}_{s} \mathrm{~d} s\right)\right\}-\left(1-\mathrm{e}^{-(1+c T)}\right) \ell(0) \\
& =\int_{0}^{T} \mathrm{e}^{-\left(T^{-1}+c\right) t} \mathrm{~d} \overline{\mathscr{M}}_{t}+\int_{0}^{T} \mathrm{e}^{-\left(c+T^{-1}\right) t}\left\{c \bar{\ell}_{t}-\left(T^{-1}+c\right)\left(\overline{\mathscr{M}}_{t}+c \int_{0}^{t} \bar{\ell}_{s} \mathrm{~d} s\right)\right\} \mathrm{d} t \\
& \leq \quad-\left(1-\mathrm{e}^{-(1+c T)}\right) \ell(0) \\
& \leq \overline{\mathscr{M}}_{T}+\int_{0}^{T} \mathrm{e}^{-\left(c+T^{-1}\right) t}\left\{c \bar{\ell}_{t}-\left(T^{-1}+c\right)\left(\bar{\ell}_{t}-\ell(0)\right)\right\} \mathrm{d} t-\left(1-\mathrm{e}^{-(1+c T)}\right) \ell(0) \\
& \leq \overline{\mathscr{M}}_{T}-\frac{1}{T \mathrm{e}^{1+c T}} \int_{0}^{T} \bar{\ell}_{t} \mathrm{~d} t .
\end{aligned}
$$

Combining this with

$$
\mathbb{E} \mathrm{e}^{\varepsilon \overline{\mathscr{M}_{t}}} \leq \mathbb{E} \mathrm{e}^{1+\varepsilon \cdot \mathscr{M}(T)} \leq \mathrm{e}\left(\mathbb{E} \mathrm{e}^{2 \varepsilon^{2}\langle\mathscr{M}\rangle(T)}\right)^{1 / 2},
$$

we complete the proof.

C3.1 Corollary 3.2. Assume (A) and let $\left|\nabla^{(2)} W\right|^{2} \leq \delta W$ hold for some constant $\delta>0$. Then there exists a constant $c>0$ such that

$$
\begin{aligned}
& \mathbb{E}^{\xi} \exp \left[\frac{1}{2\|\sigma\|^{2} \delta T^{2} \mathrm{e}^{2+2 c T}} \int_{0}^{T}\left\|W\left(X_{t}, Y_{t}\right)\right\|_{\infty} \mathrm{d} t\right] \\
& \leq \exp \left[2+\frac{W(\xi(0))}{\|\sigma\|^{2} \delta T \mathrm{e}^{1+c T}}+\frac{r_{0}\|W(\xi)\|_{\infty}}{2\|\sigma\|^{2} \delta T^{2} \mathrm{e}^{2+2 c T}}\right], \quad T>r_{0} .
\end{aligned}
$$


Proof. By (A) and the Itô formula, there exists a constant $c>0$ such that

$$
\mathrm{d} W(X, Y)(s) \leq\left\langle\nabla^{(2)} W(X, Y)(s), \sigma \mathrm{d} B(s)\right\rangle+c\left\|W\left(X_{s}, Y_{s}\right)\right\|_{\infty} \mathrm{d} s .
$$

Let

$$
\mathscr{M}(t):=\int_{0}^{t}\left\langle\nabla^{(2)} W(X, Y)(s), \sigma \mathrm{d} B(s)\right\rangle, \quad l(t):=W(X, Y)(t),
$$

and let $\varepsilon=\left(2\|\sigma\|^{2} \delta T \mathrm{e}^{1+c T}\right)^{-1}$ such that

$$
\frac{\varepsilon}{T \mathrm{e}^{1+c T}}=2\|\sigma\|^{2} \varepsilon^{2}
$$

Then by Lemma 3.1 and $\left|\nabla^{(2)} W\right|^{2} \leq \delta W$, we have

$$
\begin{aligned}
& \mathbb{E}^{\xi} \exp \left[\frac{\varepsilon}{T \mathrm{e}^{1+c T}} \int_{0}^{T} \bar{l}_{t} \mathrm{~d} t\right] \leq \mathrm{e}^{\varepsilon l(0)+1}\left(\mathbb{E}^{\xi} \mathrm{e}^{2 \varepsilon^{2}\langle\mathscr{M}\rangle(T)}\right)^{1 / 2} \\
& \leq \mathrm{e}^{1+\varepsilon l(0)}\left(\mathbb{E}^{\xi} \mathrm{e}^{2 \varepsilon^{2}\|\sigma\|^{2} \delta \int_{0}^{T} \bar{l}_{t} \mathrm{~d} t}\right)^{1 / 2}=\mathrm{e}^{1+\varepsilon l(0)}\left(\mathbb{E}^{\xi} \mathrm{e}^{\frac{\varepsilon}{T \mathrm{e}^{1+c T}} \int_{0}^{T} \bar{l}_{t} \mathrm{~d} t}\right)^{1 / 2} .
\end{aligned}
$$

By using stopping times as in the proof of Lemma 2.1 we may assume that

$$
\mathbb{E}^{\xi} \exp \left[\frac{\varepsilon}{T \mathrm{e}^{1+c T}} \int_{0}^{T} \bar{l}_{t} \mathrm{~d} t\right]<\infty
$$

so that

$$
\mathbb{E}^{\xi} \exp \left[\frac{\varepsilon}{T \mathrm{e}^{1+c T}} \int_{0}^{T} \bar{l}_{t} \mathrm{~d} t\right] \leq \mathrm{e}^{2+2 \varepsilon l(0)} .
$$

This completes the proof by noting that

$$
\frac{1}{2\|\sigma\|^{2} \delta T^{2} \mathrm{e}^{2+2 c T}} \int_{0}^{T}\left\|W\left(X_{t}, Y_{t}\right)\right\|_{\infty} \mathrm{d} t \leq \frac{r_{0}\|W(\xi)\|_{\infty}}{2\|\sigma\|^{2} \delta T^{2} \mathrm{e}^{2+2 c T}}+\frac{\varepsilon}{T \mathrm{e}^{1+c T}} \int_{0}^{T} \bar{l}_{t} \mathrm{~d} t .
$$

Proof of Corollary 1.3. Let $v$ and $\alpha$ be given in Corollary 1.2. By the semigroup property and the Jensen inequality, we will only consider $T-r_{0} \in(0,1]$.

(1) By (1.5) and the definitions of $\alpha$ and $v$, there exists a constant $C>0$ such that

$$
\begin{aligned}
& \left|v^{\prime}(s) h_{2}(0)+\alpha^{\prime}(s)\right| \leq C 1_{\left[0, T-r_{0}\right]}(s)|h(0)|\left(\frac{1}{T-r_{0}}+\frac{\|M\|}{\left(T-r_{0}\right)^{2(k+1)}}\right), \quad s \in[0, T], \\
& |\Theta(s)| \leq C|h(0)|\left(1+\frac{\|M\|}{\left(T-r_{0}\right)^{2 k+1}}\right), \quad s \in[0, T], \\
& \left\|\Theta_{s}\right\|_{\infty} \leq C\left(\|h\|_{\infty}+\frac{\|M\| \cdot|h(0)|}{\left(T-r_{0}\right)^{2 k+1}}\right), \quad s \in[0, T] .
\end{aligned}
$$


Therefore, it follows from $(A 3)$ and $(A 4)$ that

$$
\begin{aligned}
|N(s)| \leq & C 1_{\left[0, T-r_{0}\right]}(s)|h(0)|\left(\frac{1}{T-r_{0}}+\frac{\|M\|}{\left(T-r_{0}\right)^{2(k+1)}}\right) \\
& +C\left(\|h\|_{\infty}+\frac{\|M\| \cdot|h(0)|}{\left(T-r_{0}\right)^{2 k+1}}\right)\left\|W\left(X_{s}, Y_{s}\right)\right\|_{\infty}^{l}
\end{aligned}
$$

holds for some constant $C>0$. Combining this with Theorem 1.1 we obtain

$$
\begin{aligned}
\left|\nabla_{h} P_{T} f(\xi)\right| \leq & C \sqrt{P_{T} f^{2}(\xi)}\left(\mathbb{E}^{\xi} \int_{0}^{T}|N(s)|^{2} \mathrm{~d} s\right)^{1 / 2} \\
\leq & C \sqrt{P_{T} f^{2}(\xi)}\left\{|h(0)|\left(1+\frac{\|M\|}{\left(T-r_{0}\right)^{2 k+1}}\right)\right. \\
& \left.+\left(\|h\|_{\infty}+\frac{\|M\| \cdot|h(0)|}{\left(T-r_{0}\right)^{2 k+1}}\right)\left(\int_{0}^{T} \mathbb{E}^{\xi}\left\|W\left(X_{s}, Y_{s}\right)\right\|_{\infty}^{2 l} \mathrm{~d} s\right)^{1 / 2}\right\}
\end{aligned}
$$

This completes the proof of (1) since due to Lemma 2.1 one has

$$
\mathbb{E}^{\xi}\left\|W\left(X_{s}, Y_{s}\right)\right\|_{\infty}^{2 l} \leq 3\|W(\xi)\|_{\infty}^{2 l} \mathrm{e}^{C s}, \quad s \in[0, T]
$$

for some constant $C>0$.

(2) By Theorem 1.1 and the Young inequality (cf. [2, Lemma 2.4]), we have

$$
\begin{aligned}
\left|\nabla_{h} P_{T} f\right|(\xi) \leq & r\left\{P_{T} f \log f-\left(P_{T} f\right) \log P_{T} f\right\}(\xi) \\
& +r P_{T} f(\xi) \log \mathbb{E}^{\xi} \mathrm{e}^{\frac{1}{r} \int_{0}^{T}\left\langle N(s),\left(\sigma^{*}\right)^{-1} \mathrm{~d} B(s)\right\rangle}, \quad r>0 .
\end{aligned}
$$

Next, it follows from (3.2) that

$$
\begin{aligned}
& \left(\mathbb{E}^{\xi} \exp \left[\frac{1}{r} \int_{0}^{T}\left\langle N(s),\left(\sigma^{*}\right)^{-1} \mathrm{~d} B(s)\right\rangle\right]\right)^{2} \leq \mathbb{E}^{\xi} \exp \left[\frac{2\left\|\sigma^{-1}\right\|^{2}}{r^{2}} \int_{0}^{T}|N(s)|^{2} \mathrm{~d} s\right] \\
& \leq \exp \left[\frac{C_{1}|h(0)|^{2}}{r^{2}}\left(\frac{1}{T-r_{0}}+\frac{\|M\|^{2}}{\left(T-r_{0}\right)^{4 k+3}}\right)\right] \\
& \quad \times \mathbb{E}^{\xi} \exp \left[\frac{C_{1}}{r^{2}}\left(\|h\|_{\infty}^{2}+\frac{\|M\|^{2}|h(0)|^{2}}{\left(T-r_{0}\right)^{4 k+2}}\right) \int_{0}^{T}\left\|W\left(X_{s}, Y_{s}\right)\right\|_{\infty}^{2 l} \mathrm{~d} s\right], \quad T \in\left(r_{0}, 1+r_{0}\right]
\end{aligned}
$$

holds for some constant $C_{1} \in(0, \infty)$. Since $2 l \in[0,1)$ and $T \leq 1+r_{0}$, there exists a constant $C_{2} \in(0, \infty)$ such that

$$
\beta\left\|W\left(X_{s}, Y_{s}\right)\right\|_{\infty}^{2 l} \leq \frac{\left(\frac{\|h\|_{\infty}^{2}}{r^{2}} \wedge 1\right)\left\|W\left(X_{s}, Y_{s}\right)\right\|_{\infty}}{2\|\sigma\|^{2} \delta T^{2} \mathrm{e}^{2+2 c T}}+C_{2} \beta^{\frac{1}{1-2 l}}\left(\frac{\|h\|_{\infty}^{2}}{r^{2}} \wedge 1\right)^{-\frac{2 l}{1-2 l}}, \beta>0
$$


Taking

$$
\beta=\frac{C_{1}}{r^{2}}\left(\|h\|_{\infty}^{2}+\frac{\|M\|^{2}|h(0)|^{2}}{\left(T-r_{0}\right)^{4 k+2}}\right),
$$

and applying Corollary [3.2, we arrive at

$$
\begin{aligned}
& \mathbb{E}^{\xi} \exp \left[\beta \int_{0}^{T}\left\|W\left(X_{s}, Y_{s}\right)\right\|_{\infty}^{2 l} \mathrm{~d} s\right] \leq \exp \left[C_{2} \beta^{\frac{1}{1-2 l}}\left(\frac{\|h\|_{\infty}^{2}}{r^{2}} \wedge 1\right)^{-\frac{2 l}{1-2 l}}\right] \\
& \times\left(\mathbb{E}^{\xi} \exp \left[\frac{1}{2\|\sigma\|^{2} \delta T^{2} \mathrm{e}^{2+2 c T}} \int_{0}^{T}\left\|W\left(X_{s}, Y_{s}\right)\right\|_{\infty} \mathrm{d} s\right]\right)^{\frac{\|h\|_{\infty}^{2}}{r^{2}} \wedge 1} \\
& \leq \exp \left[\frac{C_{3}}{r^{2}}\left\{\|h\|_{\infty}^{2}\|W(\xi)\|_{\infty}+\left(\|h\|_{\infty}^{2}+\frac{\|M\|^{2}|h(0)|^{2}}{\left(T-r_{0}\right)^{4 k+2}}\right)^{\frac{1}{1-2 l}}\left(\frac{r^{2}}{\|h\|_{\infty}^{2}} \vee 1\right)^{\frac{2 l}{1-2 l}}\right\}\right]
\end{aligned}
$$

for some constant $C_{3} \in(0, \infty)$ and all $T \in\left(r_{0}, 1+r_{0}\right]$. Therefore, the desired entropy-gradient estimate follows by combining this with (3.3) and (3.4).

(3) Let $C^{\prime}>0$ be such that $r \geq C^{\prime}\left(\|h\|_{\infty}+\frac{\|M\| \cdot|h(0)|}{\left(T-r_{0}\right)^{2 k+1}}\right)$ implies

$$
\frac{C_{1}}{r^{2}}\left(\|h\|_{\infty}^{2}+\frac{\|M\|^{2}|h(0)|^{2}}{\left(T-r_{0}\right)^{4 k+2}}\right) \leq \frac{1}{2\|\sigma\|^{2} \delta T^{2} \mathrm{e}^{2+2 c T}},
$$

so that by Corollary 3.2

$$
\begin{aligned}
& \mathbb{E}^{\xi} \exp \left[\frac{C_{1}}{r^{2}}\left(\|h\|_{\infty}^{2}+\frac{\|M\|^{2}|h(0)|^{2}}{\left(T-r_{0}\right)^{4 k+2}}\right) \int_{0}^{T}\left\|W\left(X_{s}, Y_{s}\right)\right\|_{\infty}^{2 l} \mathrm{~d} s\right] \\
& \leq\left(\mathbb{E}^{\xi} \exp \left[\frac{1}{2\|\sigma\|^{2} \delta T^{2} \mathrm{e}^{2+2 c T}} \int_{0}^{T}\left\|W\left(X_{s}, Y_{s}\right)\right\|_{\infty} \mathrm{d} s\right]\right)^{\frac{2 C_{1}\|\sigma\|^{2} \delta T^{2} \mathrm{e}^{2+2 c T}}{r^{2}}\left(\|h\|_{\infty}^{2}+\frac{\|M\|^{2}|h(0)|^{2}}{\left(T-r_{0}\right)^{4 k+2}}\right)} \\
& \leq \exp \left[\frac{C\|W(\xi)\|_{\infty}}{r^{2}}\left(\|h\|_{\infty}^{2}+\frac{\|M\|^{2}|h(0)|^{2}}{\left(T-r_{0}\right)^{4 k+2}}\right)\right]
\end{aligned}
$$

holds for some constant $C>0$. Then proof is finished by combining this with (3.3) and (3.4).

Proof of Theorem [1.5. Again, we only prove for $T \in\left(r_{0}, 1+r_{0}\right]$. Applying (2.9) to $\varepsilon=1$ and using $\log f$ to replace $f$, we obtain

$$
P_{T} \log f(\xi+h)=\mathbb{E}\left\{R^{1}(T) \log f\left(X_{T}, Y_{T}\right)\right\} \leq \log P_{T} f(\xi)+\mathbb{E}\left(R^{1} \log R^{1}\right)(T) .
$$

Next, taking $\varepsilon=1$ in (2.6) and letting $n \uparrow \infty$, we arrive at

$$
\mathbb{E}\left(R^{1} \log R^{1}\right)(T) \leq \frac{1}{2} \mathbb{E}_{\mathbb{Q}_{1}} \int_{0}^{T}\left|\sigma^{-1} \Phi^{1}(r)\right|^{2} \mathrm{~d} r .
$$


By $\left(A 3^{\prime}\right),\left(A 4^{\prime}\right)$, (3.1) and the definition of $\Phi^{1}$, we have

$$
\begin{aligned}
\left|\sigma^{-1} \Phi^{1}(s)\right|^{2} \leq & C_{1}\left\{\left\|W\left(X_{s}^{1}, Y_{s}^{1}\right)\right\|_{\infty}^{2 l}+U^{2}\left(C_{1}\|h\|_{\infty}+\frac{C_{1}\|M\| \cdot|h(0)|}{\left(T-r_{0}\right)^{2 k+1}}\right)\right\}\|h\|_{\infty}^{2} \\
& +C_{1}|h(0)|^{2}\left(\frac{1}{\left(T-r_{0}\right)^{2}}+\frac{\|M\|^{2}}{\left(T-r_{0}\right)^{4(k+1)}}\right) 1_{\left[0, T-r_{0}\right]}(s)
\end{aligned}
$$

for some constant $C_{1}>0$. Then the proof is completed by combining this with (3.5), (3.6) and Lemma 2.1 (note that $\left(X^{1}(s), Y^{1}(s)\right)$ under $\mathbb{Q}_{1}$ solves the same equation as $\left(X_{s}, Y_{s}\right)$ under $\mathbb{P})$.

\section{Discrete Time Delay Case and Examples}

In this section we first present a simple example to illustrate our main results presented in Section 1, then relax assumption (A) for the discrete time delay case in order to cover some highly non-linear examples.

Example 4.1. For $\alpha \in C\left(\left[-r_{0}, 0\right] ; \mathbb{R}\right)$, consider functional $\mathrm{SDE}$ on $\mathbb{R}^{2}$

$$
\left\{\begin{array}{l}
\mathrm{d} X(t)=-\{X(t)+Y(t)\} \mathrm{d} t \\
\mathrm{~d} Y(t)=\mathrm{d} B(t)+\left\{-\varepsilon Y^{3}(t)+Y\left(t-r_{0}\right)+\int_{-r_{0}}^{0} \alpha(\theta) X(t+\theta) \mathrm{d} \theta\right\} \mathrm{d} t
\end{array}\right.
$$

with initial data $\xi=\left(\xi_{1}, \xi_{2}\right) \in C\left(\left[-r_{0}, 0\right] ; \mathbb{R}^{2}\right)$, where $\varepsilon \geq 0$ and $n \in \mathbb{N}$ are constants. For $z=(x, y) \in \mathbb{R}^{2}$, let $W(x, y)=1+|x|^{2}+|y|^{2}$ and set $Z(z)=-y^{3}$ and $b(\xi)=\int_{-r_{0}}^{0} \alpha(\theta) \xi_{1}(\theta) \mathrm{d} \theta+$ $\xi_{2}\left(-r_{0}\right)$. By a straightforward computation one has for $x, y \in \mathbb{R}$

$$
L W(x, y)=1-2 x(x+y)-2 \varepsilon y^{2 n} \leq 3 W(x, y)
$$

and for $\xi \in C\left(\left[-r_{0}, 0\right] ; \mathbb{R}^{2}\right)$

$$
\begin{aligned}
\left\langle b(\xi), \nabla^{(2)} W(\xi(0))\right\rangle & \leq 2\left|\int_{-r_{0}}^{0} \alpha(\theta) \xi_{1}(\theta) \mathrm{d} \theta+\xi_{2}\left(-r_{0}\right)\right|\left|\xi_{2}(0)\right| \\
& \leq 2\left(1+\int_{-r_{0}}^{0} \alpha(\theta) \mathrm{d} \theta\right)\|\xi\|_{\infty}^{2} .
\end{aligned}
$$

Then conditions (A1) and (A2) hold. Next, there exists a constant $c>0$ such that for any $z=(x, y)$ and $z^{\prime}=\left(x^{\prime}, y^{\prime}\right) \in \mathbb{R}^{2}$,

$$
\left|Z(z)-Z\left(z^{\prime}\right)\right|=\varepsilon\left|y^{3}-y^{\prime 3}\right| \leq c\left|y-y^{\prime}\right|\left(\left|y^{\prime}\right|^{2}+\left|y-y^{\prime}\right|^{2}\right) .
$$

Finally, for $\xi=\left(\xi_{1}, \xi_{2}\right), \xi^{\prime}=\left(\xi_{1}^{\prime}, \xi_{2}^{\prime}\right) \in C\left(\left[-r_{0}, 0\right] ; \mathbb{R}^{2}\right)$,

$$
\left|b(\xi)-b\left(\xi^{\prime}\right)\right| \leq \sqrt{2}\left(\int_{-r_{0}}^{0}|\alpha(\theta)| \mathrm{d} \theta \vee 1\right)\left\|\xi-\xi^{\prime}\right\|_{\infty} .
$$

So, (A3) holds for $l=1$ whenever $\left|y-y^{\prime}\right| \leq 1$ and (A4) holds for any $l \geq 0$. Moreover, $\left(A 3^{\prime}\right)$ and $\left(A 4^{\prime}\right)$ hold for $U(|z|)=|z|^{2}, z \in \mathbb{R}^{2}$. Therefore, Theorem 1.1, Theorem 1.5 and Corollary 1.3 hold. 
To derive the entropy-gradient estimate and the Harnack inequality as in Corollary 1.4. we need to weaken the assumption (A). To this end, we consider a simpler setting where the delay is time discrete. Consider

$$
\left\{\begin{array}{l}
\mathrm{d} X(t)=\{A X(t)+M Y(t)\} \mathrm{d} t, \\
\mathrm{~d} Y(t)=Z(X(t), Y(t))+\tilde{b}\left(X\left(t-r_{0}\right), Y\left(t-r_{0}\right)\right) \mathrm{d} t+\sigma \mathrm{d} B(t),
\end{array}\right.
$$

with initial data $\xi \in \mathscr{C}$, where $Z, \tilde{b}: \mathbb{R}^{m+d} \rightarrow \mathbb{R}^{d}$. If we define $b(\xi)=\tilde{b}\left(\xi\left(-r_{0}\right)\right)$ for $\xi=$ $\left(\xi_{1}, \xi_{2}\right) \in \mathscr{C}$, then equation (4.2) can be written as equation (1.1). For $(x, y),\left(x^{\prime}, y^{\prime}\right) \in \mathbb{R}^{m+d}$, define the diffusion operator associated with (4.2) by

$$
\mathscr{L} W\left(x, y ; x^{\prime}, y^{\prime}\right)=L W(x, y)+\left\langle\tilde{b}\left(x^{\prime}, y^{\prime}\right), \nabla^{(2)} W(x, y)\right\rangle .
$$

T4.2 Theorem 4.2. Assume that there exist constants $\alpha, \beta, \gamma>0$ with $\beta \geq \gamma$, functions $W \in$ $C^{2}\left(\mathbb{R}^{m+d}\right)$ with $W \geq 1$ and $U \in C\left(\mathbb{R}^{m+d} ; \mathbb{R}_{+}\right)$such that for $(x, y),\left(x^{\prime}, y^{\prime}\right) \in \mathbb{R}^{m+d}$

$$
\mathscr{L} W\left(x, y ; x^{\prime}, y^{\prime}\right) \leq \alpha\left\{W(x, y)+W\left(x^{\prime}, y^{\prime}\right)\right\}-\beta U(x, y)+\gamma U\left(x^{\prime}, y^{\prime}\right) .
$$

Assume further that there exists $\nu>0$ such that for $z=(x, y), z^{\prime}=\left(x^{\prime}, y^{\prime}\right) \in \mathbb{R}^{m+d}$ with $\left|z-z^{\prime}\right| \leq 1$

$$
\left|Z(z)-Z\left(z^{\prime}\right)\right|^{2} \vee\left|\tilde{b}(z)-\tilde{b}\left(z^{\prime}\right)\right|^{2} \leq \nu\left|z-z^{\prime}\right|^{2} W\left(z^{\prime}\right) .
$$

Then for $\delta:=\left(\alpha r_{0}+1\right)\|W(\xi)\|_{\infty}+\gamma r_{0}\|U(\xi)\|_{\infty}$ and $t \geq 0$

$$
\mathbb{E}^{\xi} W(X(t), Y(t)) \leq \delta e^{2 \alpha t}
$$

and

$$
\begin{aligned}
\left|\nabla_{h} P_{T} f(\xi)\right| \leq C & \sqrt{P_{T} f^{2}(\xi)}\left\{|h(0)|\left(1+\frac{\|M\|}{\left(T-r_{0}\right)^{2 k+1} \wedge 1}\right)+r_{0}^{\frac{1}{2}}\|W(\xi)\|_{\infty}^{\frac{1}{2}}\|h\|_{\infty}\right. \\
& \left.+|h(0)| \sqrt{\delta\left(T \wedge\left(1+r_{0}\right)\right)}\left(1+\frac{\|M\|}{\left(T-r_{0}\right)^{2 k+1}}\right)\right\}
\end{aligned}
$$

for all $T>r_{0}, \xi, h \in \mathscr{C}$ and $f \in \mathscr{B}_{b}(\mathscr{C})$, where $C>0$ is some constant. If moreover there exist constants $K, \lambda_{i} \geq 0, i=1,2,3,4$, with $\lambda_{1} \geq \lambda_{2}$ and $\lambda_{3} \geq \lambda_{4}$, functions $\tilde{W} \in C^{2}\left(\mathbb{R}^{m+d}\right)$ with $\tilde{W} \geq 1$ and $\tilde{U} \in C\left(\mathbb{R}^{m+d} ; \mathbb{R}_{+}\right)$such that for $(x, y),\left(x^{\prime}, y^{\prime}\right) \in \mathbb{R}^{m+d}$

$$
\frac{\mathscr{L} \tilde{W}\left(x, y ; x^{\prime}, y^{\prime}\right)}{\tilde{W}(x, y)} \leq K-\lambda_{1} W(x, y)+\lambda_{2} W\left(x^{\prime}, y^{\prime}\right)-\lambda_{3} \tilde{U}(x, y)+\lambda_{4} \tilde{U}\left(x^{\prime}, y^{\prime}\right),
$$

then there exist constants $\delta_{0}, C>0$ such that for $r \geq \delta_{0} /\left(T-r_{0}\right)^{2 k+1}, \xi, h \in \mathscr{C}$ and positive $f \in \mathscr{B}_{b}(\mathscr{C})$

$$
\begin{aligned}
& \left|\nabla_{h} P_{T} f\right|(\xi) \leq r\left\{P_{T} f \log f-\left(P_{T} f\right) \log P_{T} f\right\}(\xi) \\
& +\frac{C P_{T} f}{2 r}\left\{|h(0)|^{2}\left(\frac{1}{\left(T-r_{0}\right) \wedge 1}+\frac{\|M\|^{2}}{\left\{\left(T-r_{0}\right) \wedge 1\right\}^{4 k+3}}\right)\right. \\
& \left.+\frac{\left(1+\|M\|^{2}\right)|h(0)|^{2}}{\left\{\left(T-r_{0}\right) \wedge 1\right\}^{4 k+2}}\left(\lambda_{2} r_{0}\|W(\xi)\|_{\infty}+\lambda_{4} r_{0}\|\tilde{U}(\xi)\|_{\infty}+K T+\log \tilde{W}(\xi(0))\right)\right\} .
\end{aligned}
$$


Proof. By the Itô formula one has for any $t \geq 0$

$$
\begin{aligned}
\mathbb{E}^{\xi} W(X(t), Y(t)) \leq & W(\xi(0))+\alpha \mathbb{E}^{\xi} \int_{0}^{t}\left\{W(X(s), Y(s))+W\left(X\left(s-r_{0}\right), Y\left(s-r_{0}\right)\right)\right\} \mathrm{d} s \\
& -\beta \mathbb{E}^{\xi} \int_{0}^{t} U(X(s), Y(s)) d s+\gamma \mathbb{E}^{\xi} \int_{0}^{t} U\left(X\left(s-r_{0}\right), Y\left(s-r_{0}\right)\right) d s \\
\leq & W(\xi(0))+\alpha \int_{-r_{0}}^{0} W(X(s), Y(s)) d s+\gamma \int_{-r_{0}}^{0} U(X(s), Y(s)) d s \\
& +2 \alpha \mathbb{E}^{\xi} \int_{0}^{t} W(X(s), Y(s)) d s \\
\leq & \delta+2 \alpha \mathbb{E}^{\xi} \int_{0}^{t} W(X(s), Y(s) d s .
\end{aligned}
$$

Then (4.5) follows from the Gronwall inequality.

By Theorem 1.1, for $T-r_{0} \in(0,1]$ and some $C>0$ we can deduce that

$$
\left|\nabla_{h} P_{T} f(\xi)\right| \leq C \sqrt{P_{T} f^{2}(\xi)}\left(\mathbb{E}^{\xi} \int_{0}^{T}|N(s)|^{2} \mathrm{~d} s\right)^{1 / 2},
$$

where for $s \in[0, T]$

$$
N(s):=\left(\nabla_{\Theta(s)} Z\right)(X(s), Y(s))+\left(\nabla_{\Theta\left(s-r_{0}\right)} \tilde{b}\right)\left(X\left(s-r_{0}\right), Y\left(s-r_{0}\right)\right)-v^{\prime}(s) h_{2}(0)-\alpha^{\prime}(s) .
$$

Recalling the first two inequalities in (3.1) and combining (4.4) yields that for some $C>0$

$$
\begin{aligned}
\left|\nabla_{h} P_{T} f(\xi)\right| \leq & C \sqrt{P_{T} f^{2}(\xi)}\left\{\left(\int_{0}^{T}\left|v^{\prime}(s) h_{2}(0)+\alpha^{\prime}(s)\right|^{2} d s\right)^{1 / 2}\right. \\
& +\left(\mathbb{E}^{\xi} \int_{0}^{T}|\Theta(s)|^{2} W(X(s), Y(s)) \mathrm{d} s\right)^{1 / 2} \\
& \left.+\left(\mathbb{E}^{\xi} \int_{0}^{T}\left|\Theta\left(s-r_{0}\right)\right|^{2} W\left(X\left(s-r_{0}\right), Y\left(s-r_{0}\right)\right) \mathrm{d} s\right)^{1 / 2}\right\} \\
\leq & C \sqrt{P_{T} f^{2}(\xi)}\left\{|h(0)|\left(1+\frac{\|M\|}{\left(T-r_{0}\right)^{2 k+1}}\right)+r_{0}^{\frac{1}{2}}\|W(\xi)\|_{\infty}^{\frac{1}{2}}\|h\|_{\infty}\right. \\
& \left.+|h(0)|\left(1+\frac{\|M\|}{\left(T-r_{0}\right)^{2 k+1}}\right)\left(\int_{0}^{T} \mathbb{E}^{\xi} W(X(s), Y(s)) \mathrm{d} s\right)^{1 / 2}\right\} .
\end{aligned}
$$

This, together with (4.5), leads to (4.6).

Due to (3.3) and (3.4) we can deduce that there exists $C>0$ such that for arbitrary $r>0$ and $T-r_{0} \in(0,1]$

$$
\begin{aligned}
& \left|\nabla_{h} P_{T} f\right|(\xi) \leq r\left\{P_{T} f \log f-\left(P_{T} f\right) \log P_{T} f\right\}(\xi) \\
& +\frac{r P_{T} f(\xi)}{2}\left\{\frac{C|h(0)|^{2}}{r^{2}}\left(\frac{1}{T-r_{0}}+\frac{\|M\|^{2}}{\left(T-r_{0}\right)^{4 k+3}}\right)+\frac{C\|h\|_{\infty}^{2}\|W(\xi)\|_{\infty} r_{0}}{r^{2}}\right. \\
& \left.+\log \mathbb{E}^{\xi} \exp \left[\frac{C\left(1+\|M\|^{2}\right)|h(0)|^{2}}{r^{2}\left(T-r_{0}\right)^{4 k+2}} \int_{0}^{T} W(X(s), Y(s)) \mathrm{d} s\right]\right\} .
\end{aligned}
$$


Moreover, since for $s \in[0, T]$

$$
\tilde{W}(X(s), Y(s)) \exp \left(-\int_{0}^{s} \frac{\mathscr{L} \tilde{W}\left(X(r), Y(r), X\left(r-r_{0}\right), Y\left(r-r_{0}\right)\right)}{\tilde{W}(X(r), Y(r))} \mathrm{d} r\right)
$$

is a local martingale by the Itô formula, in addition to $\tilde{W} \geq 1$, we obtain from (4.7) that

$$
\begin{aligned}
& \mathbb{E}^{\xi} \exp \left[\left(\lambda_{1}-\lambda_{2}\right) \int_{0}^{T} W(X(s), Y(s)) \mathrm{d} s-\lambda_{2} r_{0}\|W(\xi)\|_{\infty}\right] \\
& \leq \mathbb{E}^{\xi} \exp \left[\int_{0}^{T}\left(\lambda_{1} W(X(s), Y(s))-\lambda_{2} W\left(X\left(s-r_{0}\right), Y\left(s-r_{0}\right)\right)\right) \mathrm{d} s\right] \\
& \leq \mathbb{E}^{\xi} \exp \left[K T-\int_{0}^{T} \frac{\mathscr{L} \tilde{W}\left(X(s), Y(s) ; X\left(s-r_{0}\right), Y\left(s-r_{0}\right)\right)}{\tilde{W}(X(s), Y(s))} \mathrm{d} s\right. \\
& \left.\quad-\lambda_{3} \int_{0}^{T} \tilde{U}(X(s), Y(s)) \mathrm{d} s+\lambda_{4} \int_{0}^{T} \tilde{U}\left(X\left(s-r_{0}\right), Y\left(s-r_{0}\right)\right) d s\right] \\
& \leq \exp \left(\lambda_{4} r_{0}\|\tilde{U}(\xi)\|_{\infty}+K T\right) \\
& \quad \times \mathbb{E}^{\xi}\left[\tilde{W}(X(T), Y(T)) \exp \left(-\int_{0}^{T} \frac{\mathscr{L} \tilde{W}\left(X(s), Y(s) ; X\left(s-r_{0}\right), Y\left(s-r_{0}\right)\right)}{\tilde{W}(X(s), Y(s))} \mathrm{d} s\right)\right] \\
& \leq \exp \left(\lambda_{4} r_{0}\|\tilde{U}(\xi)\|_{\infty}+K T\right) \tilde{W}(\xi(0)) .
\end{aligned}
$$

Combining (4.9) and (4.10), together with the Hölder inequality, yields (4.8).

The next example shows that Theorem 4.2 applies to the equation (4.2) with a highly non-linear drift.

Ex4.2 Example 4.3. Consider delay SDE on $\mathbb{R}^{2}$

$$
\left\{\begin{array}{l}
\mathrm{d} X(t)=-\{X(t)+Y(t)\} \mathrm{d} t \\
\mathrm{~d} Y(t)=\mathrm{d} B(t)+\left\{-Y^{3}(t)+\frac{1}{4} Y^{3}\left(t-r_{0}\right)+\frac{1}{2} X(t)-Y(t)\right\} \mathrm{d} t
\end{array}\right.
$$

with initial data $\xi \in C\left(\left[-r_{0}, 0\right] ; \mathbb{R}^{2}\right)$. In this example for $z=(x, y), z^{\prime}=\left(x^{\prime}, y^{\prime}\right) \in \mathbb{R}^{2}$ let $Z(z)=\frac{1}{2} x-y-y^{3}$ and $b\left(z^{\prime}\right)=\frac{1}{4} y^{\prime 3}$. For $W(x, y)=1+x^{2}+y^{4}$ it is easy to see that

$$
\begin{aligned}
\mathscr{L} W\left(x, y ; x^{\prime}, y^{\prime}\right) & =-2 x(x+y)+4 y^{3}\left(\frac{1}{2} x-y-y^{3}+\frac{1}{4} y^{\prime 3}\right) \\
& \leq-x^{2}+y^{2}-4 y^{4}-4 y^{6}+y^{3} y^{\prime 3}+2 y^{3} x \\
& \leq y^{2}-4 y^{4}-\frac{5}{2} y^{6}+\frac{1}{2} y^{\prime 6} .
\end{aligned}
$$

Then (4.3) holds for $\beta=\frac{5}{2}, \gamma=\frac{1}{2}$ and $U(x, y)=y^{6}$. Moreover for $z=(x, y), z^{\prime}=\left(x^{\prime}, y^{\prime}\right) \in \mathbb{R}^{2}$ there exists $c>0$ such that

$$
\left|Z(z)-Z\left(z^{\prime}\right)\right|^{2} \vee\left|b(z)-b\left(z^{\prime}\right)\right|^{2} \leq c\left|z-z^{\prime}\right|^{2}\left(\left|y-y^{\prime}\right|^{4}+\left|y^{\prime}\right|^{4}\right) .
$$


Thus condition (4.4) holds, Therefore, by Theorem 4.2 we obtain (4.6).

To derive (4.8), we take $w(x, y)=\frac{1}{4}\left(x^{2}+y^{4}\right)+\frac{1}{10} x y$ and set $\tilde{W}(x, y)=\exp (w(x, y)-\inf w)$. Compute for $\left(x, y, x^{\prime}, y^{\prime}\right) \in \mathbb{R}^{4}$

$$
\begin{aligned}
\frac{\mathscr{L} \tilde{W}}{\tilde{W}}\left(x, y, x^{\prime}, y^{\prime}\right)= & \mathscr{L} \log \tilde{W}(x, y)+\frac{1}{2}\left|\partial_{y} \log \tilde{W}\right|^{2}(x, y) \\
\leq & -\left(\frac{1}{2} x+\frac{1}{10} y\right)(x+y)+\left(y^{3}+\frac{1}{10} x\right)\left(\frac{1}{2} x-y-y^{3}+\frac{1}{4} y^{\prime 3}\right)+\frac{3}{2} y^{2} \\
& \quad+\frac{1}{2}\left(y^{3}+\frac{1}{10} x\right)^{2} \\
\leq & 0.5\left((0.35)^{2} / \epsilon+1.4\right)^{2}-(0.2325-\epsilon) x^{2}-0.5 y^{4}-0.175 y^{6}+0.1375 y^{6},
\end{aligned}
$$

where $\epsilon>0$ is some constant such that $0.2325-\epsilon>0$. Then condition (4.7) holds. Therefore, by Theorem 4.2 we obtain (4.8), which implies the Harnack inequality as in Corollary 1.4 according to [5, Proposition 4.1].

\section{References}

[1] M. Arnaudon, A. Thalmaier, F.-Y. Wang, Harnack inequality and heat kernel estimates on manifolds with curvature unbounded below, Bull. Sci. Math. 130(2006), 223-233.

[2] M. Arnaudon, A. Thalmaier, F.-Y. Wang, Gradient estimates and Harnack inequalities on non-compact Riemannian manifolds, Stoch. Proc. Appl. 119(2009), 3653-3670.

[3] G. Da Prato, M. Röckner, F.-Y. Wang, Singular stochastic equations on Hilbert spaces: Harnack inequalities for their transition semigroups, J. Funct. Anal. 257 (2009), 9921017.

[4] A. Es-Sarhir, M.-K. v. Renesse, M. Scheutzow, Harnack inequality for functional SDEs with bounded memory, Electron. Commun. Probab. 14 (2009), 560-565.

[5] A. Guillin, F.-Y. Wang, Degenerate Fokker-Planck equations : Bismut formula, gradient estimate and Harnack inequality, arXiv: 1103.2817v2.

[6] W. Liu, Harnack inequality and applications for stochastic evolution equations with monotone drifts, J. Evol. Equ. 9 (2009), 747-770.

[7] W. Liu, F.-Y. Wang, Harnack inequality and strong Feller property for stochastic fast diffusion equations, J. Math. Anal. Appl. 342(2008), 651-662.

[8] S.-X. Ouyang, Harnack inequalities and applications for multivalued stochastic evolution equations, to appear in Inf. Dimen. Anal. Quant. Probab. Relat. Topics.

[9] S.-X. Ouyang, M. Röckner, F.-Y. Wang, Harnack inequalities and applications for Ornstein-Uhlenbeck semigroups with jump, to appear in Potential Anal. 
[10] M. Röckner, F.-Y. Wang, Log-Harnack inequality for stochastic differential equations in Hilbert spaces and its consequences, Infin. Dimens. Anal. Quant. Probab. Relat. Topics 13(2010), 27-37.

[11] T. Seidman, How violent are fast controls? Mathematics of Control Signals Systems, 1(1988), 89-95.

[12] M. K. R. Scheutzow, M. K. von Renesse, Existence and uniqueness of solutions of stochastic functional differential equations, Random Oper. Stoch. Equ. 18 (2010), 267284

[13] F.-Y. Wang, Logarithmic Sobolev inequalities on noncompact Riemannian manifolds, Probab. Theory Relat. Fields 109(1997), 417-424.

[14] F.-Y. Wang, Harnack inequality and applications for stochastic generalized porous media equations, Ann. Probab. 35(2007), 1333-1350.

[15] F.-Y. Wang, Harnack inequalities on manifolds with boundary and applications, J. Math. Pures Appl. 94(2010), 304-321.

[16] F.-Y. Wang, Harnack inequality for SDE with multiplicative noise and extension to Neumann semigroup on non-convex manifolds, to appear in Ann. Probab. Available online arXiv:0911.1644.

[17] F.-Y. Wang, Derivative formula and Harnack inequality for jump processes, arXiv:1104.5531.

[18] F.-Y. Wang, J.-L. Wu and L. Xu, Log-Harnack inequality for stochastic Burgers equations and applications, to appear in J. Math. Anal. Appl. arXiv:1009.5948v1.

[19] F.-Y. Wang, C. Yuan, Harnack inequalities for functional SDEs with multiplicative noise and applications, to appear in Stoch. Proc. Appl. arXiv:1012.5688.

[20] F.-Y. Wang, L. Xu, Derivative formula and applications for hyperdissipative stochastic Navier-Stokes/Burgers equations, to appear in Inf. Dim. Quant. Probab. Relat. Topics arXiv:1009.1464.

[21] F.-Y. Wang, X.-C. Zhang, Derivative formulae and applications for degenerate diffusion semigroups, arXiv1107.0096.

[22] T.-S. Zhang, White noise driven SPDEs with reflection: strong Feller properties and Harnack inequalities, Potential Anal. 33 (2010),137-151.

[23] X.-C. Zhang, Stochastic flows and Bismut formulas for stochastic Hamiltonian systems, Stoch. Proc. Appl. 120(2010), 1929-1949. 